\title{
THE
}

\section{BRITISH JOURNAL OF SURGERY}

\section{INTRODUCTORY.}

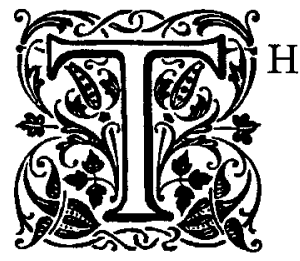

$\mathrm{HE}$ accident of being for the moment President of the Royal College of Surgeons of England, gives me the opportunity and the honour of writing the first words of this new venture in surgical journalism; of introducing it to the world; advancing its claims; and endeavouring to justify its existence.

Some apology is perhaps called for in these days, when the surfeit of medical literature placed before the enquiring student almost destroys his appetite by its very abundance. But if justification is needed, it may be found in the fact that, while in all other countries in both hemispheres where the study of surgery is most active there are special journals devoted to the subject, in Great Britain alone the progress of surgical thought and enterprise is for the most part only recorded in publications which embrace the whole subject of medicine, and whose pages are largely occupied with general matters of purely local interest. In such surroundings they may well escape the serious notice of our foreign brethren. And yet we are all brothers working hand in hand for the advancement of our Science. But our opportunities, methods of study, habits of thought, and, above all, our modes of expression, are widely different. It is therefore of vital importance that interchange of thought should be made easy, in order that we may all benefit as far as possible by the experience of others, and that our ideas may have the opportunity of acquiring fresh vigour and perhaps yielding a richer harvest by being transplanted into a new, if not a more fertile, soil. 
The intention of those who are responsible for this Journal is that it shall include the best, and as far as may be, only the best, British surgical work of the day. It will contain original articles, comments, reviews, and criticisms; and a confident appeal is made to our colleagues, not only in the Mother Country, but in India and the Colonies, to give us their hearty support, and thus to make it worthy of a place amongst the Centralblatts, Archives, and Annals with which we are familiar. With equal confidence a welcome is anticipated from our foreign contemporaries. We look back with justifiable pride to our great English surgeons of the past, and forward with hope that we may maintain our position amongst the advanced guard of surgical investigators, an assurance which is fortified by possessing in the BRITISH JOURNAL OF SURGERY a medium through which we can make our voice intelligibly heard.

RICKMAN J. GODLEE. 